The following paper posted here is not the official IEEE published version. The final published version of this paper can be found in the Proceedings of the ICON 2007: IEEE International Conference on Networks (15th : 2007 : Adelaide, Australia):pp.89-94

Copyright (c) 2007 IEEE.

Personal use of this material is permitted. However, permission to reprint/republish this material for advertising or promotional purposes or for creating new collective works for resale or redistribution to servers or lists, or to reuse any copyrighted component of this work in other works must be obtained from the IEEE. 


\section{An Architecture for IEEE 802.16 MAC Scheduler Design}

\author{
Tze Wei Tang \\ School of Electrical \& Electronic Engineering \\ The University of Adelaide \\ South Australia, 5005 \\ Email: tze.w.tang@adelaide.edu.au
}

\author{
David Green, Michael Rumsewicz and Nigel Bean \\ School of Mathematical Sciences \\ The University of Adelaide \\ South Australia, 5005 \\ Email: \{david.green, michael.rumsewicz, nigel.bean\}@ adelaide.edu.au
}

\begin{abstract}
The scheduling algorithm for IEEE 802.16 broadband wireless access system has been left open in the standard. In this paper, we consider three criteria that we have identified as important criteria for an $\mathbf{8 0 2 . 1 6}$ scheduler: Service Type differentiation, dynamic sub-frame partition and Subscriber Station differentiation. We investigate the scheduler design from a general perspective, based on these three criteria.
\end{abstract}

\section{INTRODUCTION}

Broadband Wireless Access (BWA) systems have become an important research topic as BWA emerges as a feasible technology for last mile Internet access. The main reasons for this interest stem from potential advantages, such as coverage, cost, speed of deployment and data-rate as compared with other competitor technologies.

The BWA system standard developed by the IEEE is called the IEEE 802.16 standard[1]. An 802.16 system consists of a Base Station(BS) and a number of Subscriber Stations (SS's), where each SS can represent a single user or multiple users. Two operational modes are defined in the standard: pointto-multipoint (PMP) and mesh. In PMP mode, the SS's are allowed to communicate with the BS only, whereas in mesh mode, the SS's are allowed to communicate with the BS and other SS's. In this paper, we will consider 802.16 systems operating in PMP mode.

802.16 systems utilise connection orientated data transmission, with the BS being responsible for scheduling data transmission in both the Uplink (UL) and Downlink (DL) directions. There are two grant mechanisms defined in the 802.16 standard for allowing access to the transmission medium: Grant per Connection (GPC) and Grant per Subscriber Station (GPSS). One of the benefits of using GPC is to reduce the complexity of SS's. This mode can be useful if each SS is a single user, (i.e. small number of connections per SS). However, GPSS is more scalable and efficient in terms of bandwidth utilisation, since less information is sent to the BS.

In this paper, we examine the MAC layer scheduler design problem, or more specifically, the question of how transmission opportunities should be assigned beyond traditional concepts of QoS support.

To the best of our knowledge, this is the first paper that has simultaneously considered allocating transmission opportunities according to Service Type, Transmission Type (Uplink /
Downlink) and Subscriber Station. Further, we describe the scheduler design problem from a general perspective rather than with a specific design goal in mind. That is, rather than proposing a specific algorithm, we discuss alternative objectives that schedulers may try to achieve and then discuss the associated optimisation problems that need to be solved to achieve those objectives. By using this approach, we provide the network manager with the flexibility to configure their own system to suit their needs, for example, for a network manager that has decided to build a system that treats all users equally, fairness is an important factor in his scheduler design. On the other hand, another network manager interested in maximising revenue may choose to provide Subscriber Station differentiation and thus trade away fairness amongst subscribers.

The rest of the paper is organised as follows. Section II describes the motivation of this paper and presents a discussion of earlier work. Section III gives a brief description of the current 802.16 features. Section IV describes our proposed approach in designing the BS scheduler, followed by some examples in Section V. Finally, Section VI concludes the paper and presents our further work directions.

\section{Motivation}

There are three criteria that we identify as important for an 802.16 system scheduler: Service Type (ST) differentiation, Dynamic sub-frame allocation and SS differentiation, which we now describe.

1) ST differentiation: The 802.16e standard [2] describes 5 Service Type (ST's) to enable differentiation between traffic with different QoS requirements. These will be described in detail in Section III-B. In the literature, several scheduler schemes have been proposed to achieve ST differentiation, such as Weighted Fair Queue (WFQ), Weighted Round Robin (WRR), Deficit Round Robin (DRR) and Earliest Deadline First (EDF).

2) Dynamic sub-frame partition: The $802.16 \mathrm{e}$ standard allows the proportion of UL and DL sub-frames to be dynamic. Hence, the size of UL and DL sub-frames can be varied on a per-frame basis to achieve better system performance. For instance, when there is more 
high priority traffic on the UL direction, the UL subframe can be increased accordingly. The DL and UL scheduling processes need be carried out jointly if this is to be achieved. However, most of the current work has overlooked this aspect of the standard.

3) SS differentiation: Since an 802.16 system is a centralised wireless network, it is easy to provide SS differentiation compared to a distributed wireless network. To the best of our knowledge, there is no proposed scheduler which has considered SS differentiation. There are significant potential advantages in supporting SS differentiation to provide different priorities of SS's. For instance, a commercial service provider may wish to provide better service to SS's who are willing to pay more for access. In all earlier work, the proposed 802.16 systems only provide Air-Time fairness, that is, all SS's are treated equally in some sense. When SS differentiation is provided, the 802.16 systems must also consider the SS factor, as well as the ST factor, in dynamically partitioning the sub-frames.

We have examined earlier works ([3], [4], [5], [6], [7], [8], [9], [10], [11], [12]) according to their support for these criteria. A summary of the findings is shown in Table I, where a tick indicates that the relevant criterion is explicitly incorporated in the schemes analysed in the referenced work.

TABLE I

EARLIER WORK COMPARISON

\begin{tabular}{|c|c|c|c|}
\hline Paper & Criterion 1 & Criterion 2 & Criterion 3 \\
\hline$[3]$ & $\sqrt{ }$ & $X$ & $X$ \\
\hline$[4]$ & $\sqrt{ }$ & $X$ & $X$ \\
\hline$[5]$ & $\sqrt{ }$ & $\sqrt{ }$ & $X$ \\
\hline$[6]$ & $\sqrt{ }$ & $\sqrt{ }$ & $X$ \\
\hline$[8]$ & $\sqrt{ }$ & $X$ & $X$ \\
\hline$[9]$ & $\sqrt{ }$ & $X$ & $X$ \\
\hline$[10]$ & $\sqrt{ }$ & $X$ & $X$ \\
\hline$[11]$ & $\sqrt{ }$ & $X$ & $X$ \\
\hline$[12]$ & $\sqrt{ }$ & $X$ & $X$ \\
\hline
\end{tabular}

From Table I, it is clear that the focus of the earlier work is on providing ST differentiation.

It is also seen from Table I that most of the early work has over-looked the importance of dynamically partitioning the sub-frames. In [5], the authors propose a simple algorithm for partitioning the sub-frames based on aggregate traffic condition, without considering the different ST's. On the other hand, the authors in [6] propose a dynamic UL and DL partitioning scheme which gives higher priority to the DL subframe. Giving higher priority to the DL sub-frame may not be appropriate in certain cases, especially when the system is fully loaded.

None of the earlier work has considered SS differentiation. There are many possible methods for implementing SS differentiation and in this paper, we will propose a general scheme to support SS differentiation and explain the factors that may influence scheme selection. Rather than proposing a specific algorithm, we discuss alternative objectives that schedulers may try to achieve and then discuss the associated optimisation problems that need to be solved to achieve those objectives. By using this approach, we provide the network manager with the flexibility to configure their own system to suit their needs and thus differentiate themselves from their competitors.

\section{802.16 SYSTEM DESCRIPTION}

\section{A. 802.16 PHY Layer Overview and MAC Layer Structure}

The 802.16 standard supports 4 Physical (PHY) Modes: WirelessMAN-SC (Single Carrier), WirelessMAN-SCa, WirelessMAN-OFDM (Orthogonal Frequency Division Multiplex) and WirelessMAN-OFDMA (Orthogonal Frequency Division Multiple Access). The modulation scheme includes BPSK (Binary Phase Shift Keying), QAM16 (Quadrature Amplitude Modulation) and QAM64 with different channel codings. The different modulation schemes and supported channel codings provide the multi-rate feature in the 802.16 system, enabling different data transfer rates according to the physical environment experienced by the SS and/or the technology supported by the SS.

The IEEE 802.16 standard supports both Time Division Duplexing (TDD) and Frequency Division Duplexing (FDD). One of the advantages of TDD over FDD is the flexibility of allocating the UL and DL resource. In this paper, we consider an 802.16 system that operates in TDD mode. In TDD, the data transmission is carried out using a time slot mechanism, that is, the data transmission time period is sub-divided into physical time slots. The structure of a MAC frame in TDD mode is as shown in Figure 1. For illustration purposes, the MAC frame is not drawn to scale.

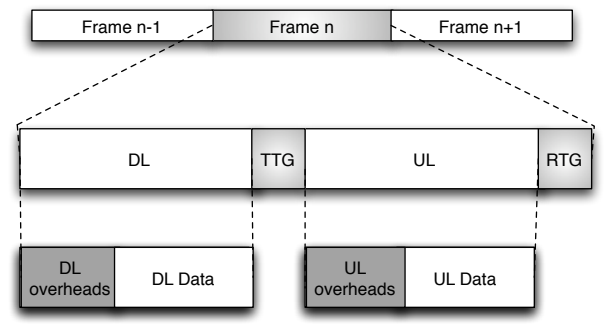

Fig. 1. Structure of a MAC frame.

In TDD, a MAC frame is divided into DL and UL subframes, which are not overlapping. There are safe guarding periods called TTG (transmit-receive transition gap) and RTG (receive-transmit transition gap) between the sub-frames. At the beginning of each of these sub-frames, there is a time period allocated for fixed overhead: DL and UL overhead. The DL overhead includes processing time for creating the DL-MAP and UL-MAP and sending these MAPs to all the SS's. These MAPs are used to store information about the transmission allocation of a MAC frame to different SS's and Service Types. All transmission activities of the 802.16 system will be based on these MAPs. The time period allocated for creating the MAPs is relatively small. Hence, it has negligible effect on the throughput of the system. It can be 
totally removed if the hardware supports parallel processing. Similarly, the UL overhead includes time for Initial Ranging (IR) and Bandwidth Request (BR). The BR period is further divided into mini-slots for bandwidth polling.

In the following, we assume that the total length of a MAC frame is of fixed length, say $P$ milliseconds with fixed overheads (DL and UL overheads) of length $Q$ milliseconds. If we divide the term, $(P-Q)$ by the slot period, $p$, which is constant for all the Physical (PHY) Modes in 802.16 systems, we obtain the total number of slots available for data transmission in a MAC frame, $M$.

The main idea of scheduling in 802.16 systems is to assign slots to a SS for either DL or UL data transmission of a particular Service Type. The scheduling process is carried out in the BS on a per-frame basis. The output of the scheduling process will be broadcast to all SS's in the form of the DL-MAP and UL-MAP. The MAP's contain the information detailing what transmission should be made in each slot in the current MAC frame.

For each of the slots, the BS decides the PHY Mode, SS, Service Type (ST) and Transmission Type (TT) (either DL or UL) involved. The PHY Mode used is chosen from the PHY Modes supported by each SS. The PHY Mode supported by a SS is determined by the Signal-to-Noise Ratio (SNR) in the Physical Layer.

\section{B. QoS Provision}

There are 5 Service Type (ST's) defined in the IEEE 802.16e standard[2], as follows:

1) Unsolicited Grant Service (UGS): This ST is designed to support real-time traffic flows that generate fixed-size data packets on a periodic basis. For example, Voice over Internet Protocol (VoIP) without Silence Suppression, Constant Bit Rate (CBR) and T1/E1 services. UGS traffic will be offered fixed-size grants on a periodic basis, without SS's needing to poll for bandwidth.

2) Real-time Polling Service (rtPS): This ST is designed to support real-time traffic flows that generate variable-size data packets on a periodic basis, such as MPEG video streaming. rtPS depends on unicast polling opportunities to request bandwidth from the $\mathrm{BS}$ and requires more overhead than UGS.

3) Extend-real-time Polling Service (ertPS): This ST offers the advantages of both UGS and rtPS. It is designed to support real-time traffic flows whose bandwidth requirements changes with time. ertPS traffic will be offered grants on a periodic basis but can also make use of unicast polling opportunities to request more bandwidth.

4) Non-real-time Polling Service (nrtPS): This ST is designed to support delay-tolerant traffic flows with variable-size data packets on a regular basis. SS's depend on broadcast polling (contention polling) opportunities to request bandwidth. Even though nrtPS is allowed to make use of unicast polling opportunities, these opportunities are very rare, compared to the unicast polling opportunities for rtPS or ertPS.
5) Best Effort Service (BE): This ST is designed for traffic flows with minimum QoS support. This service type makes use of broadcast polling opportunities only for requesting bandwidth.

All ST's need to poll for bandwidth, except UGS. In this paper, we only consider ST's that poll for bandwidth, that is, the rtPS, ertPS, nrtPS and BE.

\section{PROPOSED APPROACH}

The way that a BS allocates slots can be treated as an optimisation problem. The BS scheduler takes inputs (for example, the PHY mode active for each SS, requests for UL bandwidth from each SS for each ST, and DL bandwidth based on packets awaiting transmission to each SS for each ST), carries out some processes attempting to achieve a desired outcome with respect to bandwidth allocation, and finally produces the DL-MAP and UL-MAP. This is shown in Figure 2.

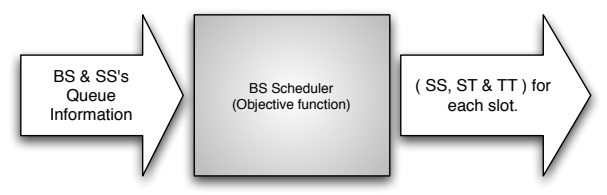

Fig. 2. Input and output of a BS scheduler.

The specific required input for the BS scheduler is the queue information from the BS and SS's, in the form of number of bits requested for each ST, TT and SS. This information is gathered in the BS either by direct access to the queues on the $\mathrm{BS}$ or by the Bandwidth Request mechanism for information from the SS's. The objective function defines the goals of the system in terms of the management priorities.

The output of the scheduler is the set of attached values for each of the slots available. These attached values are the SS, ST and TT for each of the slots.

As discussed earlier, we propose that scheduling within the 802.16 MAC layer should simultaneously take into account bandwidth requests according to ST (as done by all proposed schedulers), UL / DL (as suggested in some earlier work), and the SSs themselves (not previously proposed, to our knowledge).

Including SS as a differentiator for MAC layer access enables, for example, a charging policy to determine the priority of an SS. Furthermore, achieving fairness between SS's will not necessarily be considered a fundamental "philosophy" of the system. A flexible scheduling mechanism will enable the service provider to determine the major criteria of interest (for example, their definition of a reasonable blend of fairness, revenue and throughput). However, fairness among Service Type (ST) and Transmission Type (TT) can still be considered.

We now define the notation to be used in the remainder of this paper and then describe the basic system constraints. The following section will then describe a series of optimisation problems aimed at achieving a range of potential system goals. 


\section{A. Notation}

The terms used in this section are listed below:

$M$ : total number of data transmission slots in a MAC frame. $s_{i j k}$ : number of allocated slots for ST $i, \mathrm{SS} j$ and TT $k$ in a MAC frame.

$x_{i j k}$ : number of requested slots for ST $i, \mathrm{SS} j$ and TT $k$ in a MAC frame.

$T_{i j k}$ : throughput of ST $i$, SS $j$ and TT $k$ in a MAC frame.

$r_{j}$ : number of Bits transmitted per slot for SS $j$.

$N_{S T}$ : number of ST's (default value of 5).

$N_{S S}$ : number of SS's.

$N_{T T}$ : number of TT's (default value of 2, i.e. UL and DL). $p$ : slot period.

\section{B. Constraints}

We are interested in defining a general optimisation problem aimed at achieving the goals of the service provider in defining a MAC scheduler. However, there are a number of constraints imposed by the system itself. In particular, we enforce the obvious constraints on the total number of slots in a MAC frame, and the bounds on individual slot allocations.

$$
\begin{gathered}
\sum_{i=1}^{N_{S T}} \sum_{j=1}^{N_{S S}} \sum_{k=1}^{N_{T T}} s_{i j k} \leq M, \\
s_{i j k} \leq x_{i j k},
\end{gathered}
$$

In 802.16 systems, the multi-rate feature is supported. Hence, $r_{j}$ is defined as the number of bits transmitted in a slot for SS $j$. We assume that the $r_{j}$ value is fixed and is presumably dependent on the distance of the static SS $j$ from the BS. Therefore,

$$
T_{i j k}=\frac{r_{j} s_{i j k}}{p M} .
$$

\section{Potential Objective Functions}

In this section, we discuss some examples based on the architecture that we have formulated. Our approach here is to use a series of examples illustrating the different objectives that may be achieved using a generalised MAC scheduler, and where possible, explicitly formulating the corresponding optimisation problem. Via these examples we demonstrate the rich array of possibilities our generalised scheme can support.

In order to compare the output of these objectives, in terms of throughput and revenue of the system, we set up a test environment where we have 8 traffic flows on the UL of an 802.16 systems, as shown in Table II. The traffic flow can be tagged as High $(\mathrm{H})$ or Low (L) flows (PHY Modes), and RealTime (RT) or non-Real-Time (nRT) traffic flows. Gold (G) and Silver (S) classes are introduced to provide SS differentiation in which the Gold flows are those which are willing to pay more money for better services (applies to Objective D only).

For simplicity, the payloads for High and Low PHY modes are assumed to be 200 and 100 bits per slot respectively. We also introduce a simple charging scheme which is based on the number of bits transmitted. The charge is 2 units per bit transmitted, regardless of the class of the flow.

In this experiment, the total number of available slots is 24 in the UL of a MAC frame, while the total number of requested slots from the 8 flows is 32 (4 from each flow). Hence, the objective of the system will determine the allocation of the 24 available slots.

\section{A. Maximising System Throughput}

In this case, possibly the simplest case, we seek to maximise the total throughput of the system irrespective of ST, SS, and TT. That is, we seek to maximise:

$$
\sum_{i=1}^{N_{S T}} \sum_{j=1}^{N_{S S}} \sum_{k=1}^{N_{T T}} \frac{r_{j} s_{i j k}}{p M},
$$

or equivalently,

$$
\sum_{j=1}^{N_{S S}} r_{j}\left\{\sum_{i=1}^{N_{S T}} \sum_{k=1}^{N_{T T}} s_{i j k}\right\} .
$$

The maximal throughput is achieved by allocating slots to SSs with the highest transmission rate and when sufficient allocations have been made to meet the SS needs, moving to the SS with the next highest transmission rate, and so on. Clearly, such a scheme is not "fair", as low rate SSs can be blocked from transmission opportunities, but the simple goal of maximal network throughput is achieved.

\section{B. Max-min Air-time Fairness and Proportional Bit Fairness per $S S$}

This approach seeks to maximise the minimum of the number of slots allocated to SSs, regardless of SS differentiation. When the number of requested slots is greater than or equal to the number of slots to be allocated, each of the SS's will first be allocated the minimum of the number of data transmission slots requested. Unallocated slots are then assigned equally across SSs up to the next lowest requested allocation, and so on. While this approach focusses on air-time rather than throughput, the equivalence of this fairness (max-min air-time fairness) with proportional bit fairness was shown in [13].

To achieve proportional bit fairness per SS, which is equivalent to achieving max-min air-time fairness per SS[13], we seek to maximise,

$$
\prod_{j=1}^{N_{S S}}\left\{\frac{r_{j}}{p M} \sum_{i=1}^{N_{S T}} \sum_{k=1}^{N_{T T}} s_{i j k}\right\},
$$

or equivalently,

$$
\prod_{j=1}^{N_{S S}}\left\{r_{j} \sum_{i=1}^{N_{S T}} \sum_{k=1}^{N_{T T}} s_{i j k}\right\} .
$$

Under fully loaded conditions,

$$
\sum_{j=1}^{N_{S S}}\left\{\sum_{i=1}^{N_{S T}} \sum_{k=1}^{N_{T T}} s_{i j k}\right\}=M,
$$

and so the maximum is achieved when,

$$
\sum_{i=1}^{N_{S T}} \sum_{k=1}^{N_{T T}} s_{i j k}=\frac{M}{N_{S S}}, \quad \forall j .
$$


If $\sum_{i=1}^{N_{S T}} \sum_{k=1}^{N_{T T}} s_{i j k} \geq \sum_{i=1}^{N_{S T}} \sum_{k=1}^{N_{T T}} x_{i j k}$ for SS $j$, we mark SS $j$ as a "satisfied" SS, otherwise, we mark it as an "unsatisfied" SS. The unused slots from these "satisfied" SS's can be divided proportionally among the "unsatisfied" SS's using the same method.

\section{Max-min Bit Fairness}

This approach focusses on the throughput of each SS, and seeks to maximise the minimum throughput achieved by each SS, regardless of SS differentiation and independent of ST.

Max-min bit fairness can be achieved by ordering the total transmission request of each SS from smallest to highest and determining the number of slots each SS will require to achieve that volume of transmission. If the number of slots required is less than the number of slots available in the MAC frame, then all requests can be accommodated. If not, then allocate slots to give each SS transmission equal to the lowest request, subtract this from each SS's request, and repeat the process until all slots are assigned. In the case where not even the lowest transmission request can be fulfilled, assign slots based on the proportion of the smallest request that can be supported.

\section{Maximising Revenue - including SS differentiation in scheduling decisions}

In this objective, we include SS differentiation in our scheduling decision and seek to maximise revenue. We use a different charging scheme for this objective; for the Gold class users, the charge is 3 units per bit transmitted, while for the Silver class users, the charge is 1 unit per bit transmitted.

Maximised revenue can be achieved by firstly allocating slots to the Gold flows. Once all the Gold requests are accommodated, the unallocated slots are then assigned equally across the Silver flows.

\section{E. Discussion}

In Objective $\mathrm{A}$, we want to maximise the throughput of the system. Hence, the available slots will first be allocated to traffic flows which have higher PHY rate. The extra slots will then be allocated to flows with lower PHY rate. In fact the RT flows will be allocated slots ahead of the non-RT flows as the RT flows have tighter QoS requirements. In Objective $\mathrm{B}$, in order to achieve air-time fairness, the number of slots allocated to each traffic flow should be the same. Hence, 3 slots are allocated to each traffic flow. In Objective C, we are looking at achieving bit fairness among the 8 traffic flows. Hence, the data rate of each of the traffic flows should be equal, that is, 2 slots are allocated to flows with higher PHY rate; while 4 slots are allocated to flows with lower PHY rate, giving the same number of bits transmitted per MAC frame for all traffic flows.

Finally, we want to maximise the revenue of the system in Objective D. We consider the class (Gold and Silver) of the traffic flows, and since the Gold class users pay more money for their service, the system will try to satisfy all Gold traffic flows' requests first, and also try to maintain reasonable throughput, since the charging scheme is based on bits transmitted. Therefore, the Silver flows with higher PHY rate are also allocated slots.

Among the four objectives discussed, the max throughput is achieved in Objective A, Incidentally, the max throughput is also achieved in Objective D. This is partly because the charging scheme is based on the total number of bits transmitted, and partly, because of the way the basic parameters were selected. On the other hand, due to the differentiated charging policy used, maximum revenue is achieved in Objective D.

Apart from the above objectives, it may be useful to consider objectives that include the idea of a utility function [14]. Utility is a kind of user satisfaction measure. Each session has a utility function that may depend on both its DL and UL transmission rates.

Instead of maximising the throughput or achieving fairness among the users, it may be useful to maximise the sum of the utility of all users. The service provider can choose to give larger weight to Gold users in this process. Since the utility function depends on both the DL and UL, this will also affect the partitioning of the UL/DL of the MAC frame.

\section{CONCLUSION}

In this paper we have described the 802.16 MAC layer scheduling process. While past work has focussed heavily on the scheduling question as it pertains to Service Type differentiation, relatively little work has examined dynamic UL/DL bandwidth allocation. To the best of our knowledge, none of the past work in the area has formulated the scheduling process in terms of a Subscriber Station differentiation question. Explicitly including the SS as an input to the scheduling decision making process enables wireless access service providers to offer differentiated service levels to their customers, for example, as Gold/Silver/Bronze customers. This enables access providers to differentiate themselves from their competitors and therefore provides a mechanism for targeting certain types of customers.

We have proposed a simple mechanism for providing SS differentiation, as part of a generalised optimisation approach to decision making in MAC layer scheduling. We demonstrated the flexibility of our approach through an example that highlights how a service provider might achieve different throughput and revenue goals based on different objective functions that exploit Subscriber Station differentiation.

Our further work includes looking into incorporating service provider objectives into scheduler design and achieving SS differentiation in 802.16 systems.

\section{ACKNOWLEDGMENT}

The authors would like to thank the Australian Research Council, and industry partner Tenix Australia, for funding this research through Linkage Project LP0453508.

\section{REFERENCES}

[1] "IEEE Standard for Local and Metropolitan Area Networks - Part 16: Air Interface for Fixed Broadband Wireless Access Systems,' IEEE 802.162004, 2005. 
TABLE II

COMPARISON OF DIFFERENT OBJECTIVES.

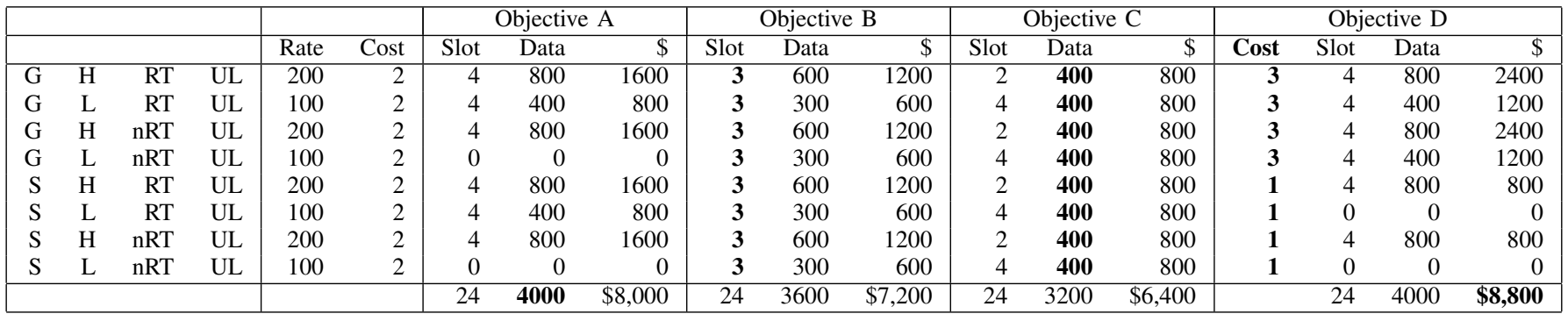

[2] "IEEE Standard for Local and Metropolitan Area Networks - Part 16: air Interface for Fixed and Mobile Broadband Wireless Access Systems- Amendment for Physical and Medium Access Control Layers for Combined Fixed and Mobile Operation in Licenced Bands," IEEE 802.16e, 2005.

[3] G. Chu, D. Wang, and S. Mei, "A QoS Architecture for the MAC Protocol of IEEE 802.16 BWA System," in Proc. IEEE Conference on Communications, Circuits and Systems, vol. 1, 2002, pp. 435-439.

[4] K. Wongthavarawat and A. Ganz, "Packet Scheduling for QoS Support in IEEE 802.16 Broadband Wireless Access Systems," in International Journal of Communication Systems, vol. 16, 2003, pp. 81-96.

[5] O. Gusak, N. Oliver, and K. Sohraby, Performance Evaluation of the 802.16 Medium Access Control Layer. Springer Berlin / Heidelberg, 2004, vol. 3280, ch. Computer and Information Sciences - ISCIS 2004, pp. $228-237$.

[6] J. Chen, W. Jiao, and H. Wang, "A Service Flow Management Strategy for IEEE 802.16 Broadband Wireless Access Systems in TDD Mode," in IEEE International Conference on Communications, vol. 5, 2005, pp. 3422-3426.

[7] N. Liu, X. Li, C. Pei, and B. Yang, "Delay Character of a Novel Architecture for IEEE 802.16 Systems," in Proc. of the Sixth International Conference on Parallel and Distributed Computing, Applications and Technologies, 2005, pp. 293-296.

[8] D. Cho, J. Song, M. Kim, and K. Han, "Performance Analysis of the IEEE 802.16 Wireless Metropolitan Area Network," in Distributed Frameworks for Multimedia Application, 2005.

[9] S. Maheshwari, "An Efficient QoS Scheduling Architecture for IEEE 802.16 Wireless MANs," Master's thesis, Indian Institute of Technology Bombay, 2005.

[10] A. Maheshwari, "Implementation and Evaluation of a MAC Scheduling Architecture for IEEE 802.16 WirelessMANs," Master's thesis, Indian Institute of Technology Kanpur, May 2006.

[11] C. Cicconetti, L. Lenzini, E. Mingozzi, and C. Eklund, "Quality of Service Support in IEEE 802.16 Networks," IEEE Network, vol. 20, no. 2, pp. 50-55, March-April 2006.

[12] A. Sayenho, O. Alanen, J. Karhula, and T. Hamalainen, "Ensuring the QoS Requirements in 802.16 Scheduling," in Proc. ACM International Symposium on Modelling Analysis and Simumation of Wireless and Mobile Systems, 2006, pp. 108-117.

[13] L. B. Jiang and S. C. Liew, "Proportional Fairness in Wireless LANs and Ad Hoc Networks," in IEEE Wireless Communications and Networking Conference, vol. 3, March 2005, pp. 1551-1556.

[14] F. Kelly, "Charging and Rate Control for Elastic Traffic," European Transactions on Telecommunications, vol. 8, pp. 33-37, 1997. 